\title{
Los talleres "epistémico-corporales" como herramientas reflexivas sobre la práctica etnográfica ${ }^{1}$
}

\author{
Marisa Ruiz Trejo ${ }^{2}$ \\ Instituto de Estudios Indígenas, \\ Universidad Autónoma de Chiapas, México \\ marisaruiztrejo@unach.mx
}

(S.) Garcia Dauder ${ }^{3}$

Universidad Rey Juan Carlos de Madrid, España

dau.dauder@urjc.es

Recibido: 5 de junio de 2017

Aceptado: 16 de mayo de 2018

Disponible en línea: 28 de diciembre de 2018

1 Este artículo de investigación se encuadra dentro de dos proyectos de investigación: "Aportes de las mujeres a las Ciencias Sociales en Chiapas y Centroamérica”, financiado por PRODEP, DSA, Dirección General de Educación Superior Universitaria y Subsecretaría de la Secretaría de Educación Pública de México, y dirigido por Marisa G. Ruiz Trejo (IEI-UNACH); y "Epistemologías feministas y activismos en salud" (FEM2016-76797-R), financiado por el Ministerio de Economía y Competitividad de España, y dirigido por Carmen Romero Bachiller (UCM).

2 Doctora en la Línea de Antropología de las migraciones, interculturalidad e identidad, Programa en Estudios Latinoamericanos, Universidad Autónoma de Madrid (España). Coordinadora de la Maestría en Estudios sobre Diversidad Cultural y Espacios Sociales e investigadora del Instituto de Estudios Indígenas, Universidad Autónoma de Chiapas.

3 Doctora en Psicología Social, Universidad Rey Juan Carlos de Madrid (España). Profesora Titular, Universidad Rey Juan Carlos de Madrid (España). 


\title{
Los talleres "epistémico-corporales" como herramientas reflexivas sobre la práctica etnográfica
}

\section{Resumen}

Los planteamientos de la antropología del sur global y las epistemologias feministas han permitido nuevas reflexiones sobre la práctica etnográfica. Desde dicho marco, proponemos los talleres como metodologías privilegiadas para la "reflexividad fuerte", colectiva y encarnada, sobre el quehacer etnográfico, no solo sobre "el saber qué", sino sobre el "saber cómo" y sobre aspectos de la investigación tradicionalmente desechados. En el artículo, conjugamos la descripción de los ejercicios de los talleres, las reflexiones colectivas surgidas de ellos, y tres artefactos teóricos para su análisis: las "prácticas corporales en investigación", la "articulación comprometida" y las "epistemologías del fuera de campo". Como resultado, analizamos el papel del cuerpo y las emociones en el proceso de investigación; las redes y vínculos que se generan, cómo y desde dónde nos comprometemos con lo que investigamos; las relaciones de poder y las violencias epistémicas en la práctica investigadora, y los dilemas, encuentros y desencuentros entre investigación y activismo.

Palabras clave: etnografia; epistemologias feministas; cuerpo; emociones; talleres; investigación activista

\section{Epistemic-corporal workshops as reflexive tools on ethnographic practice}

\begin{abstract}
The key concepts of the anthropology of the Global South and the feminist epistemologies have allowed new reflections on ethnographic practice. From this framework, we would like to propose workshops as privileged methodologies for achieving a "strong reflexivity" on the ethnographic practice that is also collective and embodied, and not just focused on knowing what, but knowing how, and on aspects of research that are traditionally discarded. In the article, we combine the descriptions of the workshop exercises, the collective reflections emerged from them, and three theoretical artifacts for their analysis: the "corporal practices in research", the "committed articulation" and the "off-field epistemologies". As a result, we analyze the role of the body and the emotions in the process of researching, the networks and bonds that are generated, the aspects involved in our commitment to these processes; the power relations and the different forms of epistemic violence in research practice; the dilemmas, the meetings and misunderstandings between research and activism.
\end{abstract}

Keywords: ethnography; feminist epistemologies; body; emotions; workshops; activist research

\section{As oficinas "epistémico-corporais" como ferramentas reflexivas sobre a prática etnográfica}

\section{Resumo}

As abordagens da antropologia do sul global e as epistemologias feministas permitiram novas reflexões sobre a prática etnográfica. Desde tal quadro, propomos as oficinas como metodologias privilegiadas para a "reflexividade forte", coletiva e encarnada sobre o quefazer etnográfico, não apenas sobre "o saber o que", mas sobre o "saber como" e sobre aspectos da pesquisa tradicionalmente descartados. No artigo, conjugamos a descrição dos exercícios das oficinas, as reflexões coletivas surgidas deles e três artefatos teóricos para sua análise: as "práticas corporais em pesquisa", a "articulação envolvida" e as "epistemologias do fora de campo". Como resultado, analisamos o papel do corpo e as emoções no processo de pesquisa; as redes e ligações geradas, como e desde onde nos comprometemos com aquilo pesquisado; as relações de poder e as violências epistémicas na prática pesquisadora; e os dilemas, encontros e desencontros entre pesquisa e ativismo.

Palavras-chave: etnografia; epistemologias feministas; corpo; emoções; oficinas; pesquisa ativista 


\section{Introducción}

En este artículo proponemos los talleres epistémico-corporales como metodologías para la reflexión colectiva y encarnada de la práctica etnográfica. Para ello, nos movemos en la intersección entre las contribuciones de las epistemologías feministas (desde diferentes contextos de producción, pero especialmente desde el sur global) y los aportes desde diferentes ejercicios de investigación activista comprometida (ya sea investigación-acción-participativa, cooperativa, co-labor, etc.). Entendemos por investigación activista, la construcción de conocimiento colectivo con una intención política explícita, al servicio de procesos críticos y de transformación social. Ello implica abordar las tensiones entre la academia comprometida y los activismos alter-anti (Leyva, 2015), pero también reconocer diferentes prácticas de conocimiento que no necesariamente pasan por lo académico o por la investigación propiamente dicha, generadas desde organizaciones de mujeres, movimientos sociales, etc. (Bastian y Berrío, 2015).

La incorporación de estos planteamientos a la antropología ha dotado a la disciplina de herramientas de intervención descolonizadora y despatriarcalizadora de la academia y del conocimiento científico -aún no legitimadas-, que permiten analizar cómo intersectan diferentes opresiones en las prácticas de investigación. Las epistemologías feministas y la investigación activista confluyen en el cuestionamiento del individualismo epistémico y de una visión no representacionista del conocimiento; en la relevancia del sujeto y la subjetividad en el proceso investigador; en la recuperación del conocimiento encarnado y experiencial y en el conocer para transformar. Estas perspectivas entienden la "objetividad" como conocimientos situados, comprometidos y responsables (Haraway, 1995) que pasan necesariamente por ejercicios de "reflexividad fuerte" sobre los procesos de investigación (Harding, 1996; Guber, 2014).

Creamos los talleres epistémico-corporales precisamente para llevar a la práctica dicha reflexividad sobre el quehacer etnográfico, como espacios colectivos que permiten la narración autobiográfica, 
pero también el diálogo intersubjetivo, ayudando a la localización del discurso y al reconocimiento de las relaciones de poder. Los talleres permiten teorizar desde las prácticas y experiencias vividas, y desde la reflexión colectiva y encarnada. Son parones o "paréntesis" en la investigación para reflexionar colectivamente sobre el "saber cómo", sobre nuestras prácticas, cuerpos y emociones en la investigación, sobre las relaciones de poder, sobre los conflictos y tensiones sobre la posición de quien investiga. En ese sentido, rompen silencios y soledades en procesos de investigación, generan conocimiento y a veces tienen incluso efectos "catárticos".

Tres artefactos teórico-políticos nos han ayudado a conceptualizar los talleres: "prácticas corporales en investigación"; "articulación comprometida" y "epistemologías del fuera de campo". Las prácticas corporales en investigación refieren al valor epistémico del cuerpo, a los conocimientos encarnados; pero también sirven para pensar cómo el ejercicio investigador y académico en general intenta disciplinar y regular cuerpos (entendiendo los cuerpos desde un punto de vista fisiológico, subjetivo y social y atravesados por estructuras sociales y ejes de opresión que intersectan), de tal forma que determinados cuerpos (que se marcan como no marcados, masculinos, blancos, etc.) parecerían tener cierto privilegio epistémico, mientras otros estarían en desventaja (aunque siempre se producen resistencias). Por otro lado, la articulación comprometida nos permite imaginar otras formas de acompañar y comprometernos en la investigación. Muchos métodos y técnicas tradicionales difícilmente reflejan los "compromisos corporales" y las implicaciones emocionales que se generan en la investigación activista, a partir de los cuales se coproduce conocimiento en relación (Esteban, 2011). Con esta herramienta, no solo se pretende romper la separación rígida y desigual entre "sujeto de investigación" y "objeto de estudio", sino sugerir formas de articulación de posiciones heterogéneas, desiguales y conflictivas desde las que se genera conocimiento comprometido y responsable. Por último, las epistemologías del fuera de campo nos ayudan a pensar en los excedentes de la investigación y en las ignorancias que generan, pero que conforman el encuadre oculto de lo que se visibiliza. 
En el artículo, conceptualizaremos estos tres artefactos intentando conjugar la descripción de los ejercicios prácticos de los talleres, las reflexiones colectivas surgidas de estos y las herramientas teóricas propuestas para su análisis. En definitiva, los talleres nos posibilitan poner en práctica la reflexividad fuerte sobre la producción de conocimientos situados y crear espacios de reflexión sobre los conflictos y tensiones sobre la posición de quien investiga.

\section{Metodologias de reflexión sobre procesos}

Siguiendo los objetivos feministas de descolonizar y despatriarcalizar la academia, los talleres epistémico-corporales están orientados no solo a investigaciones académicas, sino a cualquier práctica de conocimiento generada desde la lucha social. ${ }^{4}$ Estos talleres han sido organizados en distintas universidades, congresos y centros sociales, ${ }^{5}$ dirigidos principalmente a investigadores e investigadoras que trabajan en contextos de violencia en América Latina, fundamentalmente mediante investigación activista. ${ }^{6}$ Particularmente han asistido investigadoras feministas, así como antropólogas que se dedican a la práctica etnográfica. Cabe destacar que hemos facilitado varias modalidades de talleres, algunos de dos horas, otros de cuatro y otros de quince (amoldando el formato y los ejercicios a los tiempos).

Hemos desarrollado ejercicios prácticos para cada uno de los tres artefactos teórico-metodológicos expuestos, con el objetivo general del auto-conocimiento y la reflexión colectiva. Con los talleres subrayamos la importancia del conocimiento de nuestras vivencias y experiencias -de privilegios y opresiones- como punto de partida para investigar y para la lucha política. Además, nos interesa

4 Incluidas prácticas artísticas.

5 El primero se realizó en la UNACH (Chiapas) en el año 2013. Posteriormente, en 2014, en la UAM-Madrid; varios en la "Eskalera Caracola" y en otros centros sociales de Madrid en 2015; en 2016, en el CESMECA, Chiapas, en la Facultad de Ciencias Sociales de la UNACH en el CIESAS, en Ciudad de México, así como en el CIEG, antes PUEG, de la UNAM. También organizamos otro taller en el Congreso de Ciencia, Tecnología y Género de la Universidad de Costa Rica. Uno de los más recientes se realizó en el centro Feminista "Punto Gozadera" de la Ciudad de México.

6 Utilizamos el femenino porque en todos los talleres las participantes fueron mayoritariamente mujeres. 
reflexionar sobre los cambios en procesos de investigación complejos (no sobre productos o publicaciones), y sobre lo que queda fuera o se trata como excedente en las investigaciones. Nos centramos en el papel del cuerpo, la subjetividad y las emociones en la investigación, las relaciones de poder y la posición desde la que investigamos y nos vinculamos, las tensiones entre investigación y activismo, etc., y en cómo todo ello forma parte y atraviesa los procesos de investigación, las acciones y decisiones que se llevan a cabo y la co-producción y transformación conjunta "sujeto/objeto" de estudio. Además, otro objetivo que perseguimos es el reflexionar sobre cómo cuidar a otros/as y auto-cuidarnos en los procesos de investigación.

Entendemos los talleres como espacios corporales, no se discuten lecturas, se "conceptualizan sentires" (Calla citado en Ruiz Trejo, 2014). Por ello, intercalamos ejercicios dialógicos con otros centrados en la interacción corporal, cuidando especialmente los primeros momentos de la presentación, los acuerdos previos para espacios seguros. La idea es crear un clima de confianza que abra el diálogo, la conciencia intersubjetiva y a veces al desahogo de lo no hablado. De esta forma, se convierten en espacios de intercambio donde emerge conocimiento encarnado y se discuten procesos metodológicos adaptados a compromisos. Además, son herramientas que nos ayudan a repensar formas de producción de conocimiento híbridas (alternativas a la distinción "sujeto/objeto", "experto/lego") y las diferentes prácticas de articulación con quienes se investiga.

En los siguientes apartados, presentaremos de manera más detallada los tres artefactos teórico-metodológicos, con el fin de ofrecer algunos de los aportes que hemos recuperado de los talleres organizados en distintos espacios a lo largo de estos años.

\section{Prácticas corporales en investigación}

A través del término "prácticas corporales en investigación", y siguiendo el trabajo de la antropóloga feminista Mari Luz Esteban 
(2004, p. 46), en los talleres nos interesa "hacer consciente y explícito el entrelazamiento entre la experiencia corporal propia y la investigación" (lo que ella denomina "antropología encarnada") y, de esta manera, romper con esa idea de que la producción de conocimiento pasa únicamente por la dimensión mental. Se trata de ejercicios de "reflexividad fuerte" sobre aquellas experiencias a lo largo del proceso de investigación en las que nuestro cuerpo se haya visto expuesto de una forma más consciente y relevante. Esos ejercicios corporales y reflexivos nos permiten repensar el valor epistémico del cuerpo, de los conocimientos encarnados; también de cómo se regulan y normativizan los cuerpos de la investigación y, por último, las dinámicas inter-corporales "sujeto-objeto".

Además, nos permiten analizar cómo determinados cuerpos están en ventaja/desventaja epistémica y se les atribuye mayor o menor autoridad cognitiva o legitimidad para ser escuchados. Nos referimos a cuerpos sociales atravesados por diferentes ejes de opresión (género, raza, clase, orientación sexual, diversidad funcional, etc.) y que experimentan diferentes formas de discriminación epistémica. En los talleres surgen múltiples ejemplos de mujeres investigadoras infravaloradas o tuteladas como "jóvenes" en la investigación por sus cuerpos, ya no digamos si dichas investigadoras son indígenas, cuyo conocimiento pocas veces es reconocido como experto y muchas como testimonio (Cumes, 2015). En otros casos, la "heterosexualidad obligatoria" sitúa en posiciones violentas e incómodas a aquellas/os cuyos cuerpos y deseos no se adaptan a ella (Rich, 1980).

Así pues, las posiciones de sujeto corporeizadas son epistemológicamente relevantes. No solo porque el cuerpo dota o quita legitimidad, sino porque la experiencia corporal genera un punto de vista que abre o cierra campos de ignorancia, en función de su grado de comodidad o incomodidad en el sistema social donde se encuentre (Ahmed, 2015). La posición del sujeto "mujeres" es un punto de partida privilegiado, si bien sin garantías, para plantearse preguntas de investigación sobre las desigualdades de género ( $\mathrm{y}$ a la inversa, las posiciones normativas suelen ser cegadoras). No solo eso, el propio 
enfoque epistemológico del que se parte puede "moldear" una actitud corporal. La actitud corporal del investigador positivista, que entiende la objetividad como "distancia" y "neutralidad", no puede ser la misma que la de quien entiende la investigación como articulación comprometida. La investigación también hace cuerpos en relación, desde el habitus académico-investigador, ${ }^{7}$ hasta cómo reaccionamos a un cuerpo-otro al tiempo que lo cosificamos. ${ }^{8}$ En nuestros talleres, solemos comenzar con dos ejercicios cuyo objetivo es este tipo de reflexividad sobre el propio cuerpo en la investigación: "líneas de la vida" y "los itinerarios corporales en la investigación".

La actividad "líneas de la vida" surgió como forma de implementar la "reflexividad fuerte", es decir, que el "sujeto" de investigación sea examinado en los mismos términos que el "objeto" de conocimiento (Harding, 1996). Ello implica que quien investiga teste por sí mismo/a la sensación de ser ubicado como "objeto", o bien en forma de auto-conocimiento o bien a través de otra mirada. Desde esta perspectiva, la objetividad no es posible desde una posición desencarnada que no se haya auto-observado, sin auto-conocimiento (Ruiz Trejo, 2015), al contrario, "resulta pertinente partir de una misma para conocer a los/as otros/as" (Esteban, 2004). En palabras de Audre Lorde, feminista negra lesbiana, es necesario que: "busquemos en ese lugar del conocimiento de nosotras mismas y toquemos el terror y el odio de cualquier diferencia que vive ahí. Veamos qué cara lleva. Es entonces que lo personal tanto como lo político puede empezar a iluminar todas nuestras opciones" (1988, p. 93).

En concreto, el ejercicio "líneas de la vida" consiste en la construcción de una narración biográfica, a través de la

\footnotetext{
7 Conformado por un espacio androcéntrico que genera un habitus masculino y de clase social. Muchas investigadoras aprenden a desarrollar "autoridad corporal" a través de gestos o posturas corporales determinadas (espaldas híperrectas, mentones altos, hombros hacia atrás, etc.). A otras, el habitus masculino, de clase y heterosexual les produce lo contrario: espaldas arqueadas, hombros encogidos, voz baja, manos sudorosas, etc.

8 Y ello siempre que planteemos una investigación como conocimiento de un Otro.

9 Este ejercicio es deudor de otros cursos de formación en los que hemos participado, como los organizados por el Grupo Latinoamericano de Estudio, Formación y Acción Feminista (GLEFAS), en el que Ochy Curiel y Yuderkys Espinosa enfatizan en la importancia de reflexionar sobre los propios privilegios; también los organizados por Marcela Lagarde con su insistencia en analizar las violencias pero también las posibilidades de resistencia.
} 
auto-observación y la reflexividad. Pero dicho relato debe estar guiado por la conciencia de los privilegios y las opresiones que se han experimentado (sobre estas últimas, haciendo énfasis también en las resistencias). Para ello, en los talleres damos las siguientes instrucciones: "traza una linea imaginaria de la vida, identifica violencias/opresiones que hayas vivido en diferentes momentos (con sus vulnerabilidades); reflexiona sobre las estrategias de resistencia que has adoptado; y, finalmente, identifica privilegios que has disfrutado". Posteriormente, la redacción individual es leída en grupo, en función de las necesidades y cuidados que requiera cada participante y asegurando que nadie se sienta juzgado/a. ${ }^{10}$ Por último, se hace una puesta en común de las ideas principales como parte de un proceso de construcción colectiva de conocimiento sobre las experiencias vividas.

Tras ello, generalmente en otra sesión, realizamos el ejercicio de los "itinerarios corporales" en la investigación, el cual está basado en la propuesta teórico-metodológica de Mari Luz Esteban (2004). En este caso, son itinerarios corporales escritos por investigadoras, sobre sus propios procesos de investigación (en función de los temas concretos que trabajan) y con el objeto de reflexionar sobre su "compromiso corporal" en la investigación ${ }^{11}$-siguiendo a la propia Esteban (2008)-. El objetivo es aplicar, en un ejercicio reflexivo de auto-conocimiento, la teoría corporal a la práctica investigadora (entendiendo la "investigación" en toda su extensión, no solo como el trabajo de campo). Los itinerarios no expresan linealidad, sino más bien "fragmentos" corporales significativos. ${ }^{12}$ Se trata de abordar la tensión entre el cuerpo individual, social y político, ya que si bien son narrados como procesos individuales, remiten siempre a un colectivo. Por otro lado, se analiza el cuerpo como lugar de desigualdad social pero también de empoderamiento, desde una "encarnación conflictiva, interactiva y resistente" (Esteban, 2004, pp. 47-48).

\footnotetext{
10 Tras el ejercicio de escritura, se pueden utilizar materiales creativos para complementar la línea de vida (dibujos, collage, performances, canciones, teatro, etc.).

11 Muchas veces la reflexión también sirve para el empoderamiento corporal de las investigadoras.

12 Para Esteban, los itinerarios corporales implican la paradoja de "narrar el cuerpo", por lo que recomienda la utilización complementaria de otras técnicas más "corporales".
} 
Para esta auto-reflexión sobre nuestros cuerpos en la investigación, en los talleres damos las siguientes instrucciones: "identifica escenas, momentos o situaciones concretas del proceso de investigación (o de tu trayectoria investigadora) donde tu cuerpo se haya visto expuesto o lo hayas experimentado con mayor intensidad o conciencia; descríbelo de forma detallada, priorizando en la descripción las sensaciones o prácticas corporales / físicas; escribelo en dos o tres páginas desplazándote de lo corporal a lo subjetivo y a lo social, de lo micro a lo macro, hilvanando experiencias a contextos". ${ }^{13}$ Tras la reflexión individual y la lectura en común de los itinerarios, se orienta la discusión y reflexión colectiva hacia las relaciones de poder "incorporadas" y "emocionadas" en los procesos de investigación. Con ello se relacionan los dos ejercicios: cómo se incorporan o se hacen cuerpo los privilegios y vulnerabilidades de la propia investigadora en los procesos de investigación y en la articulación comprometida con lo que/quienes se investiga.

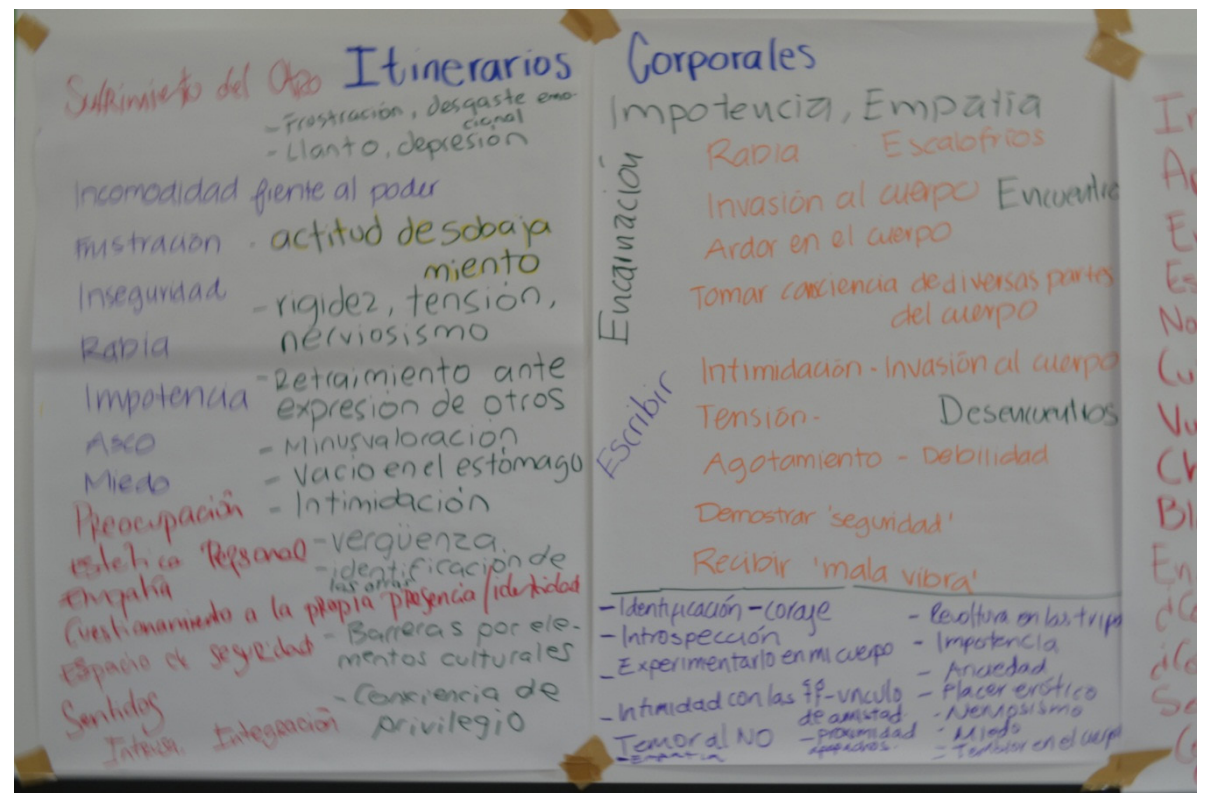

Figura 1. Taller "Prácticas corporales en Investigación",

Centro de Estudios Superiores de México y Centroamérica

${ }^{13}$ Utilizamos el texto "Transformar el silencio en lenguaje y acción" de Audre Lorde (2003) para "calentar" la voz y explicar la importancia de contar nuestras propias experiencias personales. 


\section{La articulación comprometida}

Proponemos la "articulación comprometida"14 como un artefacto teórico que alude a un particular tipo de metodología de investigación etnográfica, centrada más en procesos, prácticas y acompañamientos que en técnicas concretas y puntuales de recogida de información. Con el concepto "articulación" tratamos de romper la rígida y jerárquica distinción entre sujeto-objeto de investigación, también con visiones individualistas y representacionistas de la ciencia. ${ }^{15}$ Además, sugerimos trabajar de manera "comprometida" con quienes colaboramos en nuestras investigaciones, esto es, asumir el carácter transformador, no neutro, de la investigación.

El concepto surgió de las críticas feministas a la insuficiencia de determinadas técnicas como la entrevista o la observación participante para aprehender procesos de investigación en los que se produce conocimiento intersubjetivo o en relación; en situaciones menos concretas que darle al play de una grabadora, más diluidas en el tiempo, desde "el hacer vínculos" o el "compromiso corporal" (Esteban, 2011).

Con la articulación comprometida pretendemos cuestionar el individualismo epistémico. El conocimiento no es un producto, sino un proceso colectivo, una actividad en comunidad que surge de prácticas sociales. Por otro lado, como ya hemos dicho, no todas las posiciones son equivalentes, y poseen diferentes condiciones de posibilidad para el desarrollo de un saber aceptado o legitimado. Frente al descubridor solitario, enfatizamos la importancia de vincular las condiciones materiales de producción del conocimiento con la generación del mismo: por ejemplo, contextos de crisis y guerras y "prácticas de conocimiento otras" (Leyva, 2015).

\footnotetext{
${ }^{14}$ Este trabajo es deudor de otros usos de la "articulación” en investigación: García-Dauder y Romero Bachiller (2002), Montenegro y Pujol (2014), Cruz Contreras (2015) y Ruiz Trejo (2015).

${ }^{15}$ La visión representacionista desde la filosofía de la ciencia alude a una forma de entender el conocimiento en términos de un individuo-sujeto que representa "a modo de espejo" a un objeto. Ello implica la ausencia de valores o de cualquier implicación emocional que medie en dicho conocimiento y lo pueda distorsionar.
} 
La articulación rompe también con la idea de un sujeto autónomo de conocimiento. Frente a nociones de "objetividad estática", basadas en el distanciamiento defensivo, en el control y dominio entre sujeto-objeto, partimos de una "objetividad relacional" que hace uso de la experiencia subjetiva en la búsqueda de conocimiento y reconoce la diferencia yo-otro como una oportunidad para una relación más profunda y articulada, para afectar/se (Keller, 1991). De esta forma, se resalta el potencial epistémico de experiencias de implicación emocional o, por qué no, apasionada (Campbell, 2001): los usos de lo erótico (deseos, bailes, comidas, etc.) como fuente de poder y conocimiento (Lorde, 2003). ${ }^{16}$

Esta objetividad relacional choca, por ejemplo, con un modelo jerárquico de hacer entrevistas basado en la distinción y distanciamiento entre entrevistador-entrevistado, sujeto-objeto (convertido fácilmente en objeto-que-se-usa). Algo complicado cuando ambas partes de la comunicación comparten experiencias de vida (como muchas veces ocurre en la investigación activista). Frente a ello, la articulación implica formas de acompañamiento procesuales, conversaciones y preguntas bidireccionales, ${ }^{17}$ vulnerabilidades recíprocas y compartidas que, lejos de ser sesgos que amenazan la objetividad, abren la confianza para el conocimiento intersubjetivo y dialógico (Oakley, 1981). Cuando hablamos de implicación emocional o cuidados con lo que/con quienes se investiga, nos referimos también a la responsabilidad (en las voces o perspectivas parciales, en la escritura, etc.) ${ }^{18}$ ello implica conocer también en qué medida la investigación ha modificado sus experiencias y les ha afectado. ${ }^{19}$

\footnotetext{
${ }^{16}$ Siguiendo a Aparicio (1998) y su "mezcla de la deconstrucción con el baile", y utilizando el grito de Emma Goldman, Ruiz Trejo (2015) en su tesis doctoral sobre economía política del amor en las radios latinas afirma "si no se puede bailar no es mi investigación".

17 Entre otras, responder a la pregunta: “¿por qué te interesamos?”.

${ }_{18}$ Con sus ansiedades asociadas, llegando en ocasiones a bloqueos en la escritura o a la decisión de no escribir porque hacerlo puede causar daño.

19 Blázquez, Bustos y Restrepo (2010) analizaron, por ejemplo, el carácter transformador de sus entrevistas a mujeres académicas que, en sus retrospectivas vitales, se sorprendían al tomar conciencia de las discriminaciones de género que habían experimentado en sus trayectorias y sus propias resistencias.
} 
Con la articulación comprometida pretendemos evitar a su vez referentes o metáforas visuales -como observación- cargadas de connotaciones de distancia y representación. La promesa de objetividad a través de lo visual se ha basado en la separación sujeto-objeto, entre un individuo que percibe-conoce y un objeto que es percibido-conocido. Desde la filosofia occidental, la visión nos conecta con la verdad, el ojo como modelo del intelecto, de lo mental, disociado paradójicamente de lo corporal-sensorial-como lente pasiva y transparente de la realidad (Keller y Grontkowski, 1983)-; y, a la vez, "los ojos reguladores en las prácticas racistas y civilizatorias" (Chivalán, 2015). Además, la visión se asocia a la objetividad por su aparente "atemporalidad", como una foto fija, y su aparente "neutralidad dinámica", se puede mirar sin tocar ni ser tocado, se distancia de la acción, de la interacción, e incluso se depende de la distancia para poder ver. Algunas autoras han criticado esta lógica "masculina" y "occidental-moderna" de la visión como sentido epistémico privilegiado, en relación a otros sentidos "menores" feminizados, como el tacto (Irigaray, 1980) o la voz-oído (Esteban, 2016).

Con nuestros talleres invitamos a reflexionar sobre cómo diferiría nuestra concepción del conocimiento si nos basáramos en otras metáforas sensoriales. Por ejemplo, un conocimiento unido al sentido de la audición nos conectaría más con nociones relacionales y dialógicas de la realidad: "conocer oyendo", escuchando, preguntando (Bastian y Berrío, 2015). No solo se puede recuperar un conocimiento unido a la oralidad, sino analizar las potencialidades de las voces como representantes de diferentes posiciones de sujeto (Esteban, 2016); también, por ejemplo, saber interpretar silencios. ${ }^{20}$ Por otro lado, conocer invoca la experiencia de tocar, de la mediación corporal. A diferencia de la mirada que no requiere ser parte del mundo material, conocer-sentir mediante el tacto necesariamente implica al objeto, procesos de conocer-hacer y de saber-hacer, de combinar herramientas intelectuales con manuales

${ }^{20}$ Para una gran parte de la teoría feminista, el silencio ha estado asociado a la opresión. Sin embargo, el silencio también tiene que ser contemplado como una estrategia de resistencia ante situaciones de riesgo, peligro y vulnerabilidad, no tanto como una "ausencia", sino como una "presencia". 
(Rivera Cusicanqui, 2014), de senti-pensar (Méndez Torres et al., 2013), la conjunción de "mano, cerebro y corazón" (Rose, 1987).

Con el término articulación recuperamos el tacto pero, a su vez, el potencial de la "sinestesia epistémica", la interferencia inesperada entre unos sentidos u otros, en lugar de elegir uno como privilegiado: conocer mediante la mirada parcial, pero también de modo intersubjetivo mediante la escucha al otro, con-tacto y sensibilidad. Por ejemplo, y siguiendo a Haraway (1995), otros modelos de visión que toquen, conecten o comuniquen, mirando a los ojos, tocando con la mirada. Reclamar de esta forma la naturaleza encarnada de la vista, la visión parcial desde un cuerpo complejo y contradictorio. De ahí la responsabilidad de la mirada, de cómo miramos. Mirada y voz responden a posicionamientos en relación, un desde dónde, y dependen de reconocimientos, legitimidades y luchas de poder: una articulación de visiones parciales, desde algún lugar, y voces titubeantes en una posición de sujeto colectivo. También podemos hablar de una visión-visionaria, como esperanza en el conocimiento transformador (Haraway, 1995).

En definitiva, la articulación nos sirve para revisar y resignificar el concepto de objetividad científica, replanteando la noción de neutralidad trascendente, del "testigo modesto" auto-invisible y no marcado de la ciencia moderna (Haraway, 1999). Está ligada a la "objetividad fuerte", conocimientos situados y responsables, pero también abiertos a conexiones parciales (Haraway, 1995; Harding, 1996). Se trata de generar conocimiento, no como una forma de representación de un objeto ya dado, sino como prácticas articulatorias en las que emerge conocimiento dialógico, desde el respeto, la escucha profunda y la conciencia de pluralidad de posiciones (Leyva, 2015), aunque a veces sea una persona -o equipo- la que escriba y se articule, a su vez, con el/la lector/a.

Ello rompe con la "política semiótica de la representación" (Haraway, 1999), cuya efectividad se asienta en operaciones de distanciamiento, lo cual implica la ausencia de valores o de cualquier conexión emocional que pueda distorsionar. "Lo representado 
queda reducido al estatus permanente de recipiente de la acción, sin poder ser nunca un co-actor en una práctica articulada con otros compañeros sociales diferentes, pero vinculados" (1999, p. 138). Por ejemplo, a través de "ventriloquismos coloniales", "se secuestra la agencia de sujetos otrificados y su voz solo existe a través de la voz de otros" (Méndez Torres, 2011, p. 6).

Desde la "política semiótica de la articulación" (Haraway, 1999), el conocimiento no emana del poder de representar en la distancia, sino de formas de articulación y acompañamiento desde posiciones desiguales donde se constituyen el "sujeto-objeto" de investigación. De esta forma social y relacional, se hacen los objetos de conocimiento. La realidad o los objetos no preceden a las prácticas investigadoras, sino que son estas las que los conforman, y sus fronteras emergen de la interacción social (Mol, 2002). Frente a la idea de un "testigo modesto" u observador imparcial, las y los investigadores interfieren sobre lo investigado a la vez que lo investigan. De ahí la importancia de la reflexividad: de producir no tanto efectos de distanciamiento sujeto-objeto, sino de conexión, encarnación y responsabilidad (Haraway, 1999, p. 122).

Un ejercicio que hacemos en nuestros talleres es pedir a las investigadoras que dibujen cuál es su "objeto de estudio". En la mayoría de los casos, los "objetos" quedan expuestos como si se tratara de una foto que captura una "representación original" y casi nunca ellas mismas aparecen como parte del dibujo. Tras exponer el concepto de articulación comprometida y cómo la ciencia tradicional ha construido una separación estricta entre sujeto-objeto de estudio, preguntamos a las participantes si cambiarian en algo su creación original: el resultado es que se introducen a ellas mismas -o a sus equipos- en sus dibujos, en un ejercicio de reflexividad, y representan diferentes formas de vincularse. Con ello, queremos explicar una idea clave de las epistemologías feministas: que el sujeto de conocimiento tiene que estar en el mismo plano explicativo y de análisis que el "objeto" de conocimiento. Lejos de presuponer una distancia aséptica, la "reflexividad fuerte" (Harding, 1996) supone una participación 
comprometida por la cual el sujeto de conocimiento no se desvincula del proceso de investigación y los efectos que provoca. Por ello, más que de participación, hablamos de compromiso -corporal o emocional- con la transformación.

Articular significa alcanzar términos de acuerdo, "unir cosas, cosas espeluznantes, cosas arriesgadas, cosas contingentes" (Haraway, 1999, p. 150). Ello implica construir y reconstruir herramientas de investigación en acción conjunta, que permitan visibilizar qué parte del tejido nos ha tocado hilar (Rivera Cusicanqui, 2014); por ejemplo, construir investigación dialógica, con alianzas entre grupos organizados de mujeres e investigadoras, en las que cobra gran importancia la devolución reciproca (Bastian y Berrio, 2015). También puede implicar generar "mutualidad" y "sistemas de apoyo compartido" como alternativa a las relaciones dominantes. Por ello, la importancia de reflexionar sobre cómo nos vinculamos con lo que investigamos, desde dónde, para qué y para quién (y contra quién), así como las tensiones productivas entre academia y diversos activismos (Leyva, 2015).

Desde la investigación activista, el compromiso y la transformación social son un principio ético que requiere la construcción creativa de herramientas para generar diálogos y no otredades, conocer parcialmente, tejer hilos y conexiones entre proyectos emancipadores de búsqueda de conocimiento. También implica recoger la producción de conocimiento fuera de la investigación académica, en encuentros, asambleas, talleres, diálogos, panfletos o manifiestos, vídeos, etc. (Bastian y Berrío, 2015). Romper el dualismo entre el teorizar-escribir académico y el hacer activista. Haraway habla de "conocimientos parciales, localizables y críticos, que admiten la posibilidad de conexiones llamadas solidaridad en la política y conversaciones compartidas en la epistemologia" (1995, p. 329). La articulación de diferentes voces y puntos de vista en torno a objetivos compartidos, desde las tensiones que genera la heterogeneidad de experiencias asociadas con opresiones que intersectan. Se trata de articular estas posiciones sociales múltiples y contradictorias en cuya tensión y conflicto se produce 
un conocimiento más reflexivo y por tanto más crítico y objetivo: desde "los nudos de la sabiduría feminista" (Vargas, 2015) o, como señala Aura Cumes (2015), "crear a partir de las desventajas".

\section{Las epistemologias del fuera de campo}

El concepto "fuera de campo" tiene su origen en el cine y nos permite construir un símil de la escritura científica como cámara o mirilla que limita el espacio con el enfoque, encuadra la realidad investigadora y deja en el "fuera de campo" todo aquello -parte de la realidad también- que queda fuera del encuadre. Al mismo tiempo, el "fuera de campo" refiere a momentos más allá de lo que se considera "trabajo de campo", aquellos que no se describen ni siquiera en la metodología, pero que conforman el encuadre oculto de la misma. Es aquello invisibilizado que rodea lo visible, donde continúa la vida de los "personajes" que dejan la escena, la privacidad de las investigadoras y su entorno. Así como en el cine se intentan ocultar las huellas sobre cómo se realiza la producción (salvo en el making of o las tomas falsas), también en la publicación de la investigación científica se borran muchos elementos del proceso, que quedan como residuos epistémicos del aparataje técnico, fuera de la escritura. La diferencia es que mientras en el cine el espectador participa activamente en la recreación de lo que queda fuera -basándose en la información de lo que queda dentro, lo que se sugiere, se intuye-, en la investigación se presenta el encuadre como cerrado, se corta la imaginación de quien lee, se neutraliza el entorno. Estar fuera de campo casi nunca es neutro, conforma los valores por omisión de la ciencia, los deshechos o excedentes de la ciencia.

Una parte de las epistemologias del fuera de campo constituirian lo que Nancy Tuana (2006) ha denominado "epistemologias de la ignorancia": lo que no sabemos, o lo que ni siquiera sabemos que no sabemos; lo que no se conoce porque no importa, por negligencia; lo que no quieren que sepamos o cuando no queremos 
saber porque ello implica afectar nuestros privilegios e intereses. El fuera de campo lo constituyen muchas veces aquellos saberes producidos por sujetos en desventaja epistémica, invisibilizados como no conocimiento. En los talleres, a veces, introducimos este concepto asociado a la "ciencia sin hacer" y lo vinculamos a ejercicios reflexivos sobre lo-que-no-se-conoce en cada proceso investigador.

Pero, como decíamos, el fuera de campo no es solo la producción del "no conocimiento", de "ignorancia", sino su excedente, los deshechos de la investigación. Lo que queda fuera del proceso de escritura, de lo comunicable bajo las normas metodológicas, pero con valor de reciclaje, de conocimiento para la investigación. El ejemplo más claro en una investigación sería el trabajo reproductivo, frecuentemente ignorado, que hace posible la producción material de conocimiento por parte de un sujeto con cuerpo que, entre otras cosas, se alimenta. ${ }^{21}$ Pero no solo eso, el fuera de campo son conversaciones, confesiones, cafés, o comidas donde se guisa la confianza necesaria para generar conocimiento y se sostiene lo cotidiano. Son paseos, bailes, manifestaciones, a veces cuidar a los/as niños/as o al perro de la persona a la que se quiere entrevistar. Pero también está conformado por risas, lágrimas, silencios, indignación, vergüenzas, angustia, esperanza, decepción y dolores compartidos, todo el abanico de emociones que conforman un proceso de investigación. Son los vínculos que nos unen a las personas con quienes investigamos y luchamos, el reconocimiento de nuestros privilegios y la externalización de nuestros dilemas. En nuestros talleres, proponemos trabajar sobre las epistemologías del fuera de campo a través del análisis de cinco ejes: cuerpo, emociones, poder, colectividad y activismo, tal como exponemos a continuación.

21 ¿Quién cocina, limpia y cuida mientras alguien investiga? Remitimos, por ejemplo, al libro de Mary Gabriel (2014) sobre la invisibilización de la fuerza de trabajo, de cuidados y amor de Jenny Marx y de sus hijos para que Karl Marx pudiera escribir El capital. Un trabajo reproductivo atravesado, a su vez, por relaciones de género y clase. Pero también nos referimos al trabajo reproductivo que podemos hacer como investigadoras, cuando se cocina para quien se investiga o se colabora en las labores de alimentación con quienes trabajamos en "campo". 
El primer eje consiste en intentar identificar algún momento en el proceso de investigación donde nuestros cuerpos hayan sido más evidentes o hayamos sido más conscientes de ellos (al estilo de los itinerarios corporales antes descritos). Las reflexiones fuera de campo en torno al cuerpo intentan recuperar y recordar los sentidos, por ejemplo, el papel del tacto, del oído o del olfato en nuestra investigación o alguna situación donde estos hayan sido relevantes. Como ya hemos señalado, intentamos descentrar la vista como metáfora sensorial privilegiada de conocimiento, más incorpórea que otros sentidos, y así romper con la "anestesia epistemológica", la pérdida de sensibilidad en el conocimiento. La idea en los talleres es pensar las posibilidades de otros sentidos: por ejemplo, el tacto como sentido que rompe la distinción sujeto-objeto de conocimiento y lo vincula a un sentido más afectivo, a la sensibilidad. O cómo operan "las ideologías de la voz" como mecanismos de poder en momentos concretos de la investigación (cuando se usurpa la voz, se levanta la voz, se da voz, se escucha, se desoye, se acalla o se calla), voces en desventaja epistémica (agudas-femeninas, "con acento"-racializadas) o entendidas como representantes de conocimientos situados (Esteban, 2016; Villaverde, 2016). O la importancia de recuperar fuentes orales para contrarrestar relaciones de poder y la invisibilidad de determinadas voces en la escritura ("las mujeres han dicho más de lo que han escrito") (Bastian y Berrío, 2015). También se puede "jugar" con la "sinestesia epistemológica", el potencial de la interferencia de los sentidos, como cuando se toca con la mirada, o comprender cómo se articulan unos sentidos con otros. ${ }^{22}$ En definitiva, con este eje se pretende reflexionar-siguiendo a Spinoza- sobre "qué puede un cuerpo" en relación con el conocimiento.

\footnotetext{
22 Por ejemplo, las interferencias entre vista y oído, ejemplificadas en la piel y los acentos: cómo la voz puede modificar la percepción de una apariencia o cómo una apariencia puede modificar la percepción de una voz.
} 


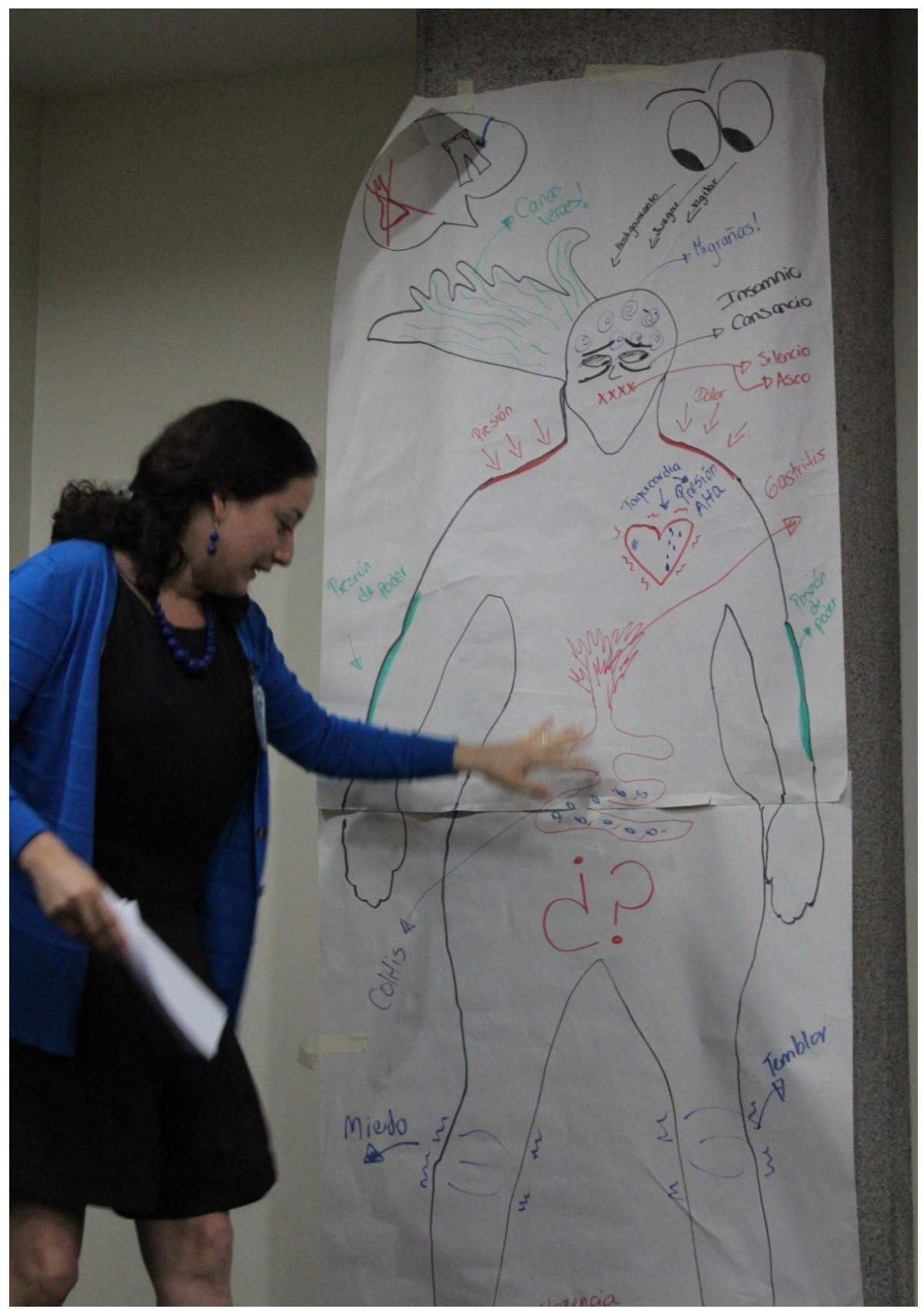

Figura 2. Taller "Experiencias corporales, comprometidas y reflexivas en investigación activista", Congreso Iberoamericano: Ciencia, Tecnología y Género: San José, Costa Rica 
Una reflexión muy poderosa que surgió en uno de los talleres que organizamos dio como resultado un rotafolio en donde las participantes dibujaron un cuerpo en el que fueron especificando algunas de las experiencias que habian vivido en sus procesos de investigación y cómo se reflejaban corporalmente. Por ejemplo, los momentos de tensión en los que se acumula carga en la espalda o algún hecho puntual en el que las participantes habían tenido miedo y les temblaban las piernas; las ojeras por el insomnio de noches de escritura, las migrañas o cólicos por estrés, contención o palabras amordazadas; más canas y dioptrias. Todas estas experiencias suelen quedar fuera de lo publicable, pero la investigadora se las lleva escritas en el cuerpo. Un dibujo como este podría ilustrar de forma original un manual de prevención de riesgos laborales para quien investiga.

Nuestro segundo eje de reflexión es la conexión entre conocimiento y emociones: las experiencias emocionales de las investigadoras o cómo nos afecta o sentimos la investigación (sentimientos hacia las participantes, miembros del equipo, hacia el tema de estudio, cómo nos afectan o afectan a la investigación, si son representadas en narrativas publicables, etc.). Una modalidad es tratar de identificar ciertas emociones o momentos emocionales que hayan estado presentes o que hayan sido relevantes en el proceso investigativo, si bien como trasfondo no publicable: por ejemplo, la vergüenza, la culpa, la rabia, el miedo, el asco, etc. Se pueden trabajar como un "viaje por las emociones" a lo largo de todo el proceso investigador (Blakely, 2007). Campbell (2001) habla de "investigaciones emocionalmente implicadas", por ejemplo, cuando se trabaja con la violencia sexual, en las que las emociones de la investigadora son tanto herramientas de investigación como fuentes de información. ${ }^{23}$

La otra modalidad consiste en centrarse en una única emoción, por ejemplo, la indignación o la vergüenza, y analizar de forma "temática" cómo ha atravesado diferentes momentos a lo largo del proceso investigador y su importancia en la generación de conocimiento. En particular, seguimos el trabajo de Sarah Ahmed (2015) sobre la politica cultural de las emociones aplicado en este caso a la investigación feminista. En sus

\footnotetext{
${ }^{23}$ A veces generando "victimizaciones secundarias" en las investigadoras, con miedos y pesadillas, por ejemplo. En otros casos, provocando de-sensibilización por saturación de violencia.
} 
palabras, el feminismo involucra una respuesta emocional "que implica una reorientación de nuestra relación corporal con las normas sociales" (259). En los talleres trabajamos con las emociones que Ahmed considera como forjadoras de vínculos feministas: la indignación frente a las injusticias, el dolor ${ }^{24}$ como efecto de diferentes formas de violencia, el amor, el deseo y la alegría en las redes con otras mujeres, el asombro que siente lo ordinario como sorprendente y la esperanza que estructura el deseo de cambio, como apertura pensable hacia lo posible. Uniendo los dos ejes de los talleres, reflexionamos también sobre cómo las emociones moldean cuerpos y objetos. Y siguiendo a la misma autora, en lo que ella denomina "sentimientos queer", abordamos el potencial epistémico de la incomodidad o desorientación y, a la inversa, el potencial de ignorancia del confort o la comodidad ${ }^{25}$ (especialmente respecto a los conocimientos encarnados).

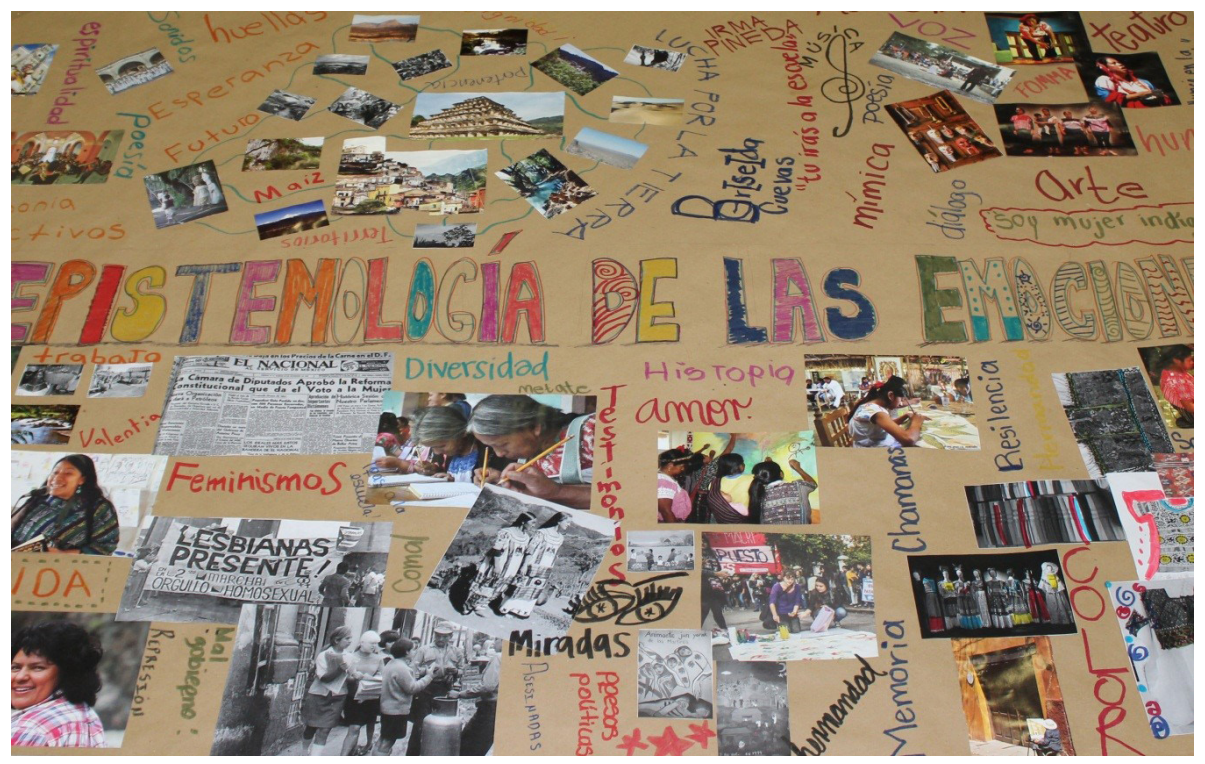

Figura 3. Taller "Cuerpo, género y feminismos", Centro de Investigaciones y Estudios de Género, UNAM-México

${ }^{24}$ María Espinosa (2016) ha analizado el papel del dolor en las etnografias: cómo se gestiona el dolor de quien habla, cuando la investigadora actúa como contenedora de dicho dolor o cuando revive el dolor que escucha. A veces, en los procesos de investigación se producen intercambios de dolores compartidos en ejercicios de vulnerabilidad recíproca que, a su vez, generan conocimiento.

${ }^{25}$ Como señala Ahmed, la normatividad es cómoda pero ciega a sus condiciones, "el confort es muy difícil de ver cuando uno lo vive" (2015, p. 227). Al contrario, desde la incomodidad o desorientación-el fuera de lugar-es mayor nuestra atención al cuerpo -y su amolde a espacios y objetos- y es más fácil reflexionar sobre las comodidades que produce el privilegio de la norma. 
El tercer eje sobre el que trabajamos las epistemologías del fuera de campo es el poder: el reconocimiento de las relaciones de poder y el diálogo sobre ellas. Para ello introducimos un ejercicio en el que las investigadoras reflexionan sobre aquellas situaciones en las que hayan podido ejercer violencia epistémica o en las que, por el contrario, hayan experimentado situaciones de vulnerabilidad en el proceso de investigación. A partir de dicho intercambio colectivo, se intentan repensar experiencias metodológicas más horizontales (desde el reconocimiento mutuo y la interlocución) y menos violentas entre investigadoras y sujetos de estudio. Ello implica especial cuidado en reconocer la heterogeneidad y tomar conciencia de con quiénes decidimos dialogar y a quiénes legitimamos (y a quiénes no) y por qué. También quién habla por quién y quién tiene autoridad para ser escuchado/a.

Por otro lado, las redes y vínculos que se crean en el proceso de investigación también son elementos importantes del fuera de campo. En los talleres, tratamos de discutir y reflexionar sobre cómo y desde dónde nos vinculamos (relacionamos, comprometemos, etc.) con quienes investigamos, cómo nuestro posicionamiento afecta al conocimiento que producimos, pero también, cómo lo que investigamos nos cuestiona. De esta forma, tratamos de establecer cómo serían las condiciones de posibilidad para una articulación comprometida, como las ya señaladas en el epígrafe anterior.

Por último, y siguiendo los postulados de las epistemologías feministas, es indispensable contemplar la transformación social en la construcción de conocimiento. Por eso, el último eje que trabajamos del "fuera de campo" tiene que ver con los dilemas políticos y éticos que quedan fuera de lo publicable en la investigación activista, pero que son una importante herramienta de conocimiento: cuando el activismo se desencuentra con la academia "en un cruce altamente tensionado" (Leyva, 2015). En este eje, trabajamos la transformación social versus la cooptación y despolitización que con frecuencia producen las instituciones universitarias; nos preguntamos a quién beneficia nuestra investigación, en qué circuitos y lenguajes circulan sus productos; cómo manejar simultáneamente 
tiempos, lenguajes y formatos académicos y activistas; cómo hacer para incorporar más experiencias, voces y perspectivas en lo académico como estrategia para transformar; y, por último, cómo hacer para no correr el riesgo de que se coopte la posibilidad de transformación social fuera del espacio académico.

\section{Reflexiones finales}

En resumen, los talleres "epistémico-corporales" permiten verbalizar y reflexionar colectivamente sobre lo que el espacio académico y sus publicaciones científicas omiten, excluyen o desechan. En ese sentido, convierten el silencio en lenguaje y acción (Lorde, 2003). Constituyen espacios seguros donde diferentes investigadoras pueden hablar de lo que no se habla, de sus procesos investigativos, de sus compromisos emocionales y corporales, de las violencias y de los cuidados. De dichos intercambios de "reflexividad fuerte" se genera mucho conocimiento, si bien deslegitimado por la investigación científica dominante.

Los talleres se convierten así en espacios de seguridad y de autocuidado, pero a su vez exponen fragilidades y vulnerabilidades, lo cual los aleja de aquellos investigadores/as resistentes a exponer a flor de piel sus subjetividades en la investigación, bajo la máscara de una aparente neutralidad, racionalidad y acorporalidad científica. Ello implicaría perder poder y tomar conciencia de cómo los privilegios marcan una investigación.

\section{Referencias}

Ahmed, S. (2015). La política cultural de las emociones. México: UNAM - PUEG.

Aparicio, F. (1998). Listening to salsa gender, Latin popular music, and Puerto Rican cultures. Hanover: University Press of New England. 
Bastian, A. I. y Berrío, L. R. (2015). Saberes en diálogo: mujeres indigenas y académicas en la construcción del conocimiento. En X. Leyva (Ed.), Prácticas otras de conocimiento (s). Entre crisis, entre guerras. Tomo II (pp. 199-222). San Cristóbal de las Casas: Retos.

Blakely, K. (2007). Reflections on the role of emotion in feminist research. International Journal of Qualitative Methods, 6(2), 59-68.

Blázquez, N., Bustos, O., Delgado, G. y Fernández, L. (2008). Mujeres académicas: entre la ciencia y la vida. En C. Miqueo, M. J. Barral y C. Magallón (Eds.), Estudios Iberoamericanos de Género en Ciencia, Tecnología y Salud (227245). Zaragoza: Prensas Universitarias de Zaragoza.

Campbell, R. (2001). Emotionally involved: The impact of researching rape. Nueva York: Routledge.

Chivalán, M. (2015). Los ojos: “reguladores” en las prácticas racistas y civilizatorias. Guatemala: Comunidad de Estudios Mayas. Recuperado de http: / / commaya2012.blogspot.com.es/2015/03/los-ojosreguladores-en-laspracticas.html

Cruz, A. (2015). Los conocimientos situados de Donna Haraway como recurso epistemológico para la investigación crítica (Tesis doctoral). UCM, Madrid.

Cumes, A. (2015). La presencia subalterna en la investigación social. En X. Leyva (Ed.), Prácticas otras de conocimiento(s). Entre crisis, entre guerras. Tomo I(135-158). San Cristóbal de las Casas: Retos.

Espinosa, M. (2016). Etnografias del dolor. II Congreso Internacional de Antropología AIBR. Universidad de Barcelona.

Esteban, M. L. (2004). Antropología del cuerpo. Barcelona: Bellaterra.

Esteban, M. L. (2008). Etnografia, itinerarios corporales y cambio social: apuntes teóricos y metodológicos. En M. Martínez (Coord.), La materialidad de la identidad (135-158). Donostia: Hariadna Editorial. 
Esteban, M. L. (2011). Crítica al pensamiento amoroso. Barcelona: Bellaterra.

Esteban, M. L. (2016). La etnografia somática y feminista: ¿cómo desestabilizar la mirada antropológica sin perder de vista las desigualdades sociales? II Congreso Internacional de Antropología AIBR. Universidad de Barcelona.

Gabriel, M. (2014). Amor y capital. Madrid: Intervención cultural.

García-Dauder, S. y Romero Bachiller, C. (2002). Rompiendo viejos dualismos: De las (im)posibilidades de la articulación. Revista Athenea Digital, (2). Recuperado de http://ddd.uab.es/pub/athdig/15788946n2a4.pdf

Guber, R. (Comp.). (2014). Prácticas etnográficas. Ejercicios de reflexividad de antropólogas de campo. Buenos Aires: IDES - Miño y Dávila.

Haraway, D. (1995). Ciencia, “cyborgs” y mujeres. Madrid: Cátedra.

Haraway, D. (1999). Las promesas de los monstruos: Una política regeneradora para otros inapropiados/bles. Politica y Sociedad, (30), 121-163.

Harding, S. (1996). Ciencia y Feminismo. Madrid: Morata.

Irigaray, L. (1980). When our lips speak together. Signs, 6(1), 69-79.

Keller, E. F. (1991). Reflexiones sobre género y ciencia. Valencia: Edicions Alfons el Magnanim.

Keller, E. F. y Grontkowski, C. R. (1983). The mind's eye. In S. Harding y M.B. Hintikka (Eds.), Discovering reality (207-224). Netherlands: Springer.

Leyva, X. (2015). ¿Academia versus activismo? Repensarnos desde y para la práctica teórica-política. En X. Leyva (Ed.), Prácticas otras de conocimiento (s). Entre crisis, entre guerras. Tomo II (199-222). San Cristóbal de las Casas: Retos.

Lorde, A. (1998). Las herramientas del amo nunca desarmarán la casa del amo. En C. Moraga y A. Castillo (Eds.), Esta puente mi espalda (89-93). San Francisco: Ism press ed. 
Lorde, A. (2003). La hermana, la extranjera. Madrid: Horas y Horas.

Méndez Torres, G. (2011). El ventriloquismo y el largo caminar de las mujeres indígenas. Seminario Virtual Internacional "Creación de Prácticas de conocimiento desde el género, los movimientos y las redes". Recuperado de http://www. encuentroredtoschiapas.jkopkutik.org/pdfs/TEXTOGEORGINAMENDEZ.pdf

Méndez Torres, G. et al. (Coord.) (2013). Senti-pensar el género. Perspectivas desde los pueblos originarios. Guadalajara: La Casa del Mago.

Mol, A. M. (2002). The body multiple: Ontology in medical practice. Durham: Duke University Press.

Montenegro, M. y Pujol, J. (2014). Investigación, articulación y agenciamientos tecnológicos de género: el caso “Generatech". Athenea digital, 14(1), 29-48.

Oakley, A. (1981). Interviewing women: A contradiction in terms. En H. Roberts (Ed.), Doing feminist research (30-61). Nueva York: Routledge.

Rich, A. (1980). Compulsory heterosexuality and lesbian existence. Signs, 5(4), 631-660.

Rivera, S. (2014). La noción de "derecho" o las paradojas de la modernidad postcolonial. En Y. Espinosa, D. Gómez y K. Ochoa (Eds.), Tejiendo de otro modo: Feminismo, epistemología y apuestas descoloniales en Abya Yala (121134). Popayán: Universidad del Cauca.

Rose, H. (1987). Hand, Brain and Heart. En S. Harding y J. O’Barr (Eds.), Sex and Scientific Inquiry (265-282). Chicago: The University of Chicago Press.

Ruiz Trejo, M. (2014). Feminismos, memoria y justicia en América Latina. Entrevista a la antropóloga feminista Pamela Calla. Programa de Radio Contratiempo (número 210). Recuperado de http: / / www.contratiempohistoria. org $/$ ? $=4459$

Ruiz Trejo, M. (2015). Amor al aire. Antropología situada de las radios latinas en Madrid (Tesis doctoral). Universidad Autónoma de Madrid, España. 
Tuana, N. (2006). The speculum of ignorance. Hypatia, 21(3), 1-19.

Vargas, V. (2015). Itinerario de los otros saberes. En X. Leyva (Ed.), Prácticas otras de conocimiento (s). Entre crisis, entre guerras. Tomo II (150-172). San Cristóbal de las Casas: Retos.

Villaverde, T. (2016). La ideología de la voz. Pikara Magazine. Recuperado de http:/ /www.pikaramagazine.com/2016/07/las-ideologias-de-la-voz/

\section{Cómo citar este artículo}

Ruiz Trejo, M. y García Dauder, (S.). (2018). Los talleres "epistémico-corporales" como herramientas reflexivas sobre la práctica etnográfica. Universitas Humanística, 86, 55-82. https://doi.org/10.11144/Javeriana.uh86.tech 